\title{
Study on microbial detection technology in food safety
}

\author{
Xindi Zhang ${ }^{1}$ \\ ${ }^{1}$ Dalian Chengze Testing CO.LTD, Dalian,116100, China
}

\begin{abstract}
Economic development has not only led to the steady development of the gross national economy, but also provided a fundamental guarantee for the life of the residents at this stage. However, with the rapid development of economy, people's attention to hidden safety problems has gradually shifted from big problems to "small details" of food safety. At the same time, in order to reduce the health problems of consumers in the process of eating products, we should start from the source of food, and use microbial technology in the current food safety testing, so as to fundamentally improve the quality of food safety. At present, PCR, impedance, ATP bioluminescence, lamp and enzyme-linked immunosorbent assay are widely used. In this paper, the role of microbial detection technology was described, and the application of microbial detection technology in food safety detection was analyzed in depth, hoping to provide a reference for ensuring food safety through the promotion of microbial detection technology.
\end{abstract}

\section{INTRODUCTION}

Nowadays, with the rapid development of science and technology, the public pay more and more attention to food safety, and one of the important indicators of food safety evaluation is food microbiology, which makes people pay more attention to microbial detection technology [1]. At present, the detection method of food microorganisms, its steps is more cumbersome, and timeconsuming, and in the face of sudden food safety incidents, its detection results will be lagging behind the regulatory needs [2]. Therefore, the rapid detection technology of food microorganisms is becoming more and more popular [3]. The following will analyze, summarize and predict the trend of popular microbial detection technologies in recent years, such as the research progress of PCR technology, impedance method, enzyme-linked immunosorbent assay, lamp, ATP bioluminescence technology, etc.

In recent years, many food safety incidents have occurred in many countries and regions. For example, on October 19, 2011, Staphylococcus aureus was detected in "missing" three fresh dumplings; on November 7 of the same year, the same pathogen was detected in Sanquan cabbage and pork dumplings; Meanwhile, on November 17 , Staphylococcus aureus was also detected in the soup of Wanchai wharf. [5]. According to relevant information, as of February 2, 2012, only in the third quarter of 2011, there were 73 food poisoning incidents reported in China, including 3224 cases of poisoning and 61 deaths [6]; among them, microbial food poisoning events accounted for $70.7 \%$ and $47.9 \%$ of the total and reported cases respectively. Nowadays, the living standard of residents is gradually improving, people's safety awareness is becoming stronger and stronger, and food safety requirements are becoming more and stricter. Food safety has become the focus of the world. The development of food microbiological inspection technology and its application in food safety inspection have also attracted more and more public attention [8].

Food safety is related to the national economy and people's livelihood. Once it is not handled properly, it will lead to serious social problems and expose fierce social contradictions [9]. Over the years, the material living standard of residents has gradually improved, and the public has gradually paid attention to food safety issues. People have found that microbial pollution is the most important factor leading to food problems, which puts forward higher requirements for food safety testing technology, but also brings greater challenges. We must take relevant measures to standardize its standards and improve microbial detection technology To ensure the safety and reliability of food [10].

\section{THE MAIN CONTENT OF FOOD MICROBIOLOGICAL DETECTION TECHNOLOGY}

\subsection{Concept of Microbial Detection Technology}

Food safety is closely related to people's life. With the improvement of the quality of life, food safety has been widely concerned. However, large-scale food poisoning incidents still occur from time to time. The food safety problem is not the self-protection and prevention of the masses, but the food production and food supervision A challenge raised by the inspection unit is to put forward more stringent requirements for food safety testing. In recent years, illegal production and "three no food" have shown signs. Under the lack of perfect legal constraints, 
food counterfeiting and selling are constantly on the rise. It can be said that it is a cancer of social stability and people's health, which has attracted the attention of relevant departments of the state and all sectors of society. With China's increasing influence in the world, food import and export trade is frequent, and food safety issues are also widely concerned in the world.

\subsection{Application of Microbial Detection Technology in Food Safety Detection}

At present, domestic food safety problems are generally exposed, and the distribution and development of food production enterprises are not very balanced. Therefore, the use of safe and efficient food detection technology can ensure the safety of food. Microbial detection technology is a relatively advanced detection technology rising now. If it is applied to food safety detection, it can ensure food to a certain extent Quality and safety indicators. Microbial detection technology can not only detect the environment of food production and processing, to see whether it conforms to the environmental standards of food hygiene, but also can quickly analyze the microbial components in food, and can also provide certain reference for health supervision departments. Food microbiological inspection technology adheres to the principle of prevention first, which shows the degree of food safety and the degree of isolation. It can not only ensure people's health needs, but also avoid economic losses, laying a solid foundation for improving food quality, and also plays an important role in ensuring food import and export safety. Microbial detection technology can be applied to every link of food safety production and the whole packaging process. The use of microbial detection technology ensures the scientific and accurate food safety production, and further ensures the quality of food production. The data obtained by using microbial detection technology for food detection is not only the basis for food safety production, but also the powerful basis for food safety management departments to formulate food safety standards and policies, so that the relevant food standards and policies are constantly improved.

\section{EXPERIMENTAL IDEAS AND DESIGN}

\subsection{Experimental Ideas}

Food microbiological detection technology is based on microbiology, using the theoretical knowledge and technical means of biosensor, modern immunology, automatic instruments, molecular biology and other aspects to study and explore the pathogens in food, especially the types, properties and quantity of pathogenic microorganisms, and then create a food microbiological detection method in line with the contemporary era. Because this method has the advantages of accuracy, simplicity, rapidity, good sensitivity and high efficiency, it can quickly detect the pathogens in food and evaluate the food hygiene quality. The research contents of food microbial detection technology mainly include: pathogen biosensor detection technology, food borne pathogen immunological detection technology, food borne pathogen metabolite inspection technology, food borne pathogen molecular biology rapid detection, food borne pathogen automatic inspection technology, food borne pathogen molecular biology rapid detection, etc.

\subsection{Experimental Design}

In the design, this paper adopts two forms: questionnaire survey and field interview. 756 different types of food were selected as survey samples. The purpose of this study is to explore the main situation of food safety detection by microbial detection technology. Through the analysis of questionnaire and field survey results, 756 kinds of food were divided into two groups. One is the experimental group, using microbial detection method; the other is the control group, using traditional detection methods. After a period of observation, we made statistics and Analysis on the safety situation of the two groups of food, and the results are shown in Table 1. According to the investigation results of two groups of food, microbial detection technology is more effective than traditional detection method.

Table 1 Analysis of food hygiene and safety under microbial detection technology

\begin{tabular}{|c|c|c|}
\hline Investigation factors & $\begin{array}{c}\text { The experimental group } \\
(\%)\end{array}$ & The control group (\%) \\
\hline $\begin{array}{c}\text { It can analyze the microbial } \\
\text { components in food }\end{array}$ & 95.1 & 35.7 \\
\hline $\begin{array}{c}\text { Can judge whether the food } \\
\text { production environment } \\
\text { conforms to the standard }\end{array}$ & 83.2 & 66.8 \\
\hline $\begin{array}{c}\text { The method is simple and } \\
\text { efficient }\end{array}$ & 78.9 & 59.7 \\
\hline The method has high sensitivity & 86.7 & 42.3 \\
\hline
\end{tabular}




\section{DISCUSSION}

\subsection{Research and Analysis of Food Safety Problems under Microbial Detection Technology}

The hidden danger of food safety in China is becoming more and more serious under the guidance of profit value. The relevant systems and measures have not yet achieved the desired implementation effect, which requires the improvement of food safety detection technology. At present, from the perspective of implementation, the application of food safety detection technology in the process of safety monitoring has been paid attention to. In terms of detection technology, food microbial detection technology can quickly detect the content and types of microorganisms in food, and improve the convenience of people's life. Therefore, microbial detection technology can be widely used in the process of food safety detection

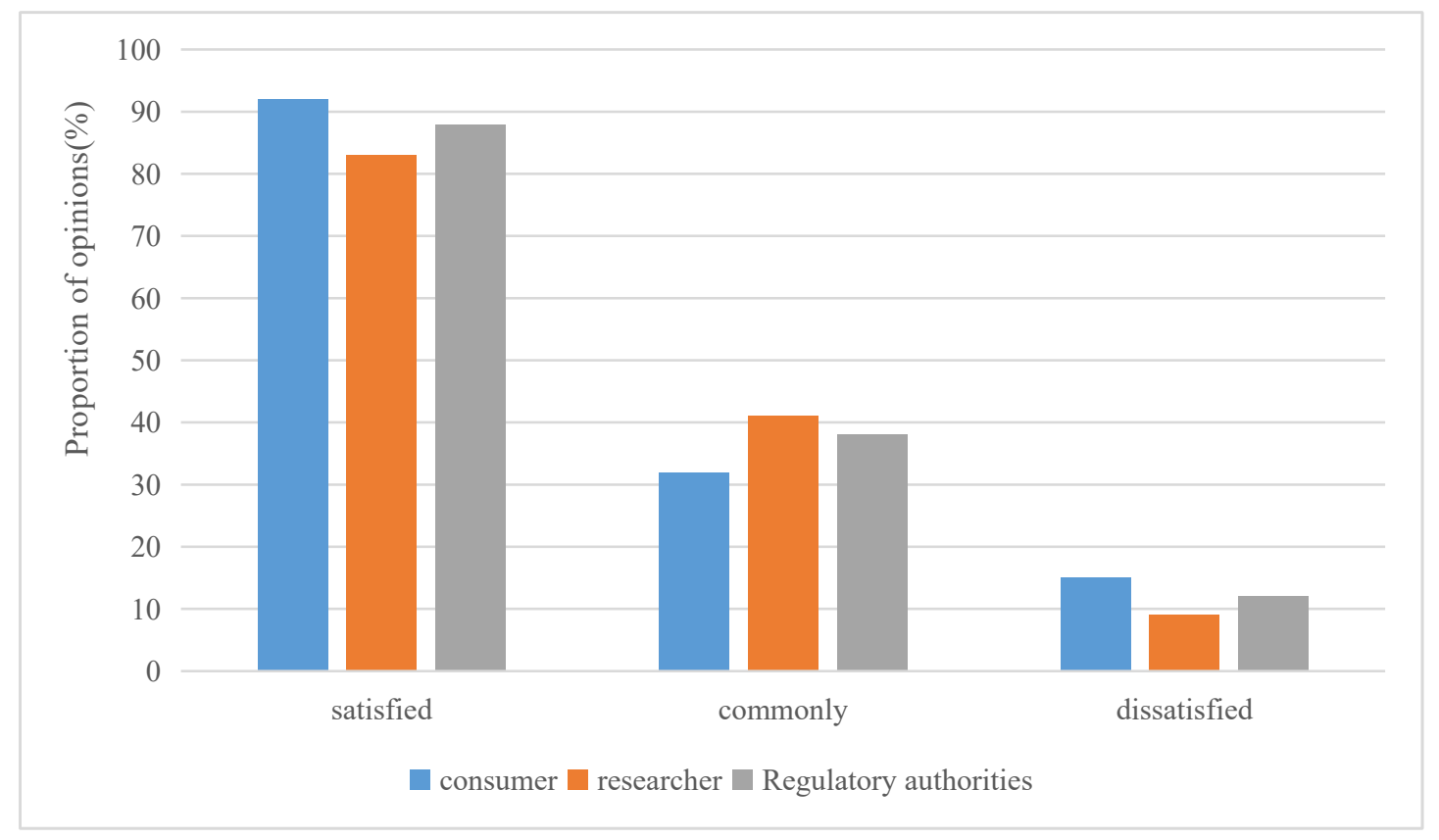

Figure 1 Survey on satisfaction of consumers, researchers and regulatory authorities on microbial detection technology

It can be seen from Figure 1 that consumers, researchers and regulatory authorities are satisfied with the use of microbial detection technology for food safety testing. In the view of consumers, microbial detection technology can make them more confident and secure to buy a variety of food. For researchers, microbial detection technology gives them a greater stage to play, can improve the accuracy and sensitivity of detection, accumulate more experience and improve the detection efficiency. For the food regulatory authorities, microbial detection technology can better ensure that the food safety indicators and quality meet the requirements, but also can provide certain reference data for it. Generally speaking, microbial detection technology makes the vast majority of people feel satisfied and can better meet the needs of the public. 


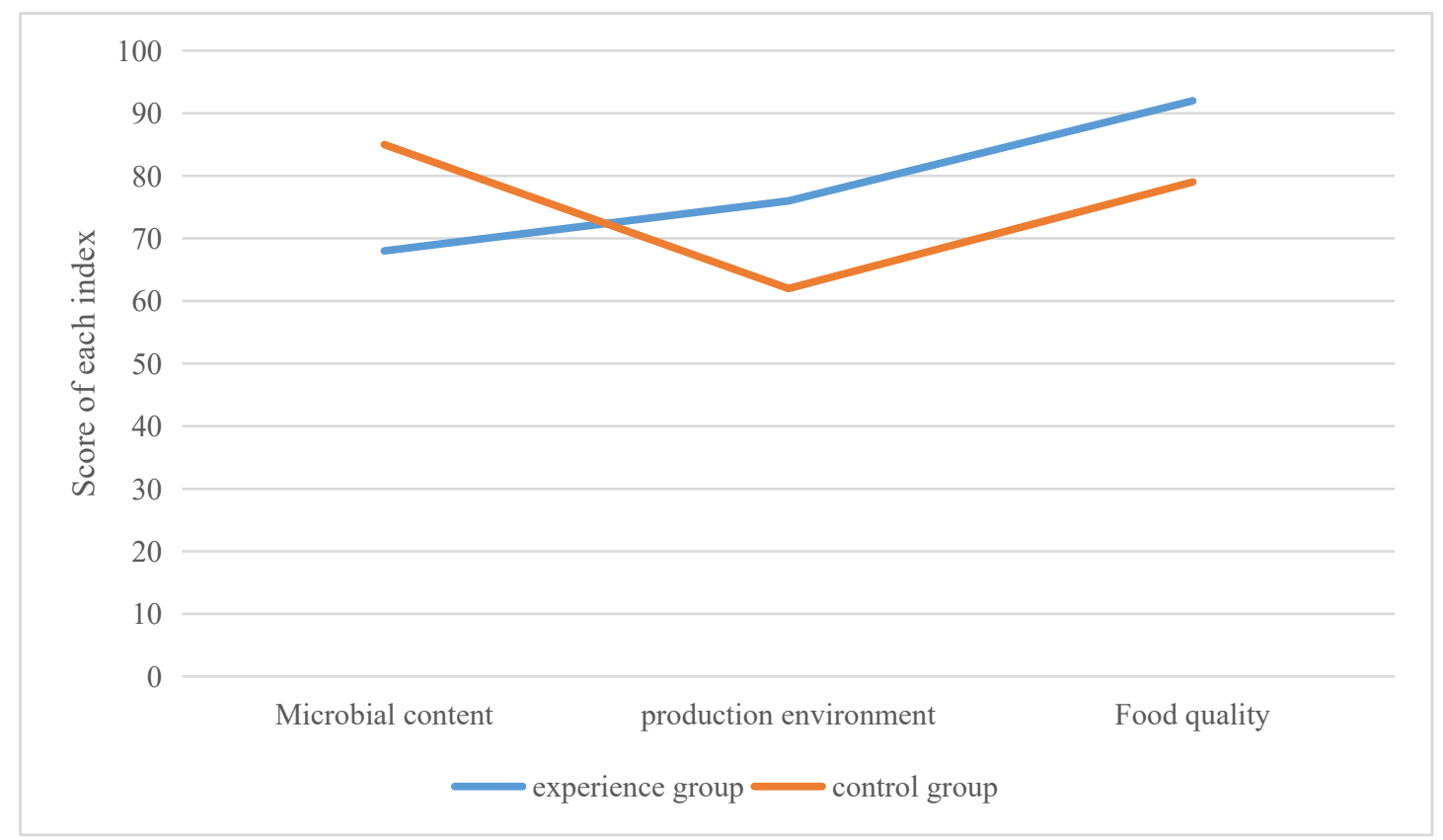

Figure 2 Analysis of various indicators after incorporating microbial detection technology into food safety

In addition, this paper further investigated the situation of various indicators after integrating microbial detection technology into food safety, and the results are shown in Figure 2. It can be seen from Figure 2 that the indicators of food after using microbial detection technology are significantly better than those of traditional detection mode. Food testing researchers have good detection ability and can cultivate their own skills. The regulatory authorities also believe that the advantages of microbiological detection technology will always outweigh the disadvantages. They think that microbial detection technology is necessary to add a layer of insurance on food safety issues.

\subsection{Suggestions on Food Safety Combined with Microbial Detection Technology}

\subsubsection{Microbial detection technology and the necessity of implementation}

Nowadays, under the guidance of profit value, the hidden danger of food safety in China is more and more obvious, and the relevant systems and measures have not yet achieved the desired implementation effect, which requires the improvement of food safety detection technology. At present, from the perspective of implementation, the application of food safety detection technology in the process of safety monitoring has been paid attention to. In terms of detection technology, food microbial detection technology can quickly detect the content and types of microorganisms in food, and improve the convenience of people's life. Therefore, microbial detection technology can be widely used in the process of food safety detection. Microbial detection can improve the safety and reliability of food to the greatest extent, and promote the safety of food production. Relevant departments need to master the appropriate detection technology to improve the efficiency of food quality inspection.

\subsubsection{The application status of microbial detection technology in food safety detection}

Nowadays, microbial detection technology has been fully used in food safety detection. The main detection technologies are nucleic acid probe technology and biosensor technology. Nucleic acid probe technology is to detect whether there are Escherichia coli and Staphylococcus aureus in food, and then quickly detect the types and quantity of these microorganisms in food. However, these need professional personnel to operate, so the operation cost is relatively high. Therefore, the application scope of microbiological detection technology is relatively small, only in the laboratory. Biosensor technology is to locate and monitor the position of microorganisms, which is mainly detected by optical fiber immune device. However, according to the specific implementation, this technology has not been reasonably used.

\subsubsection{Application and development of microbial detection technology in food safety}

According to the emerging technology is one of the most important characteristics of food microbiological detection technology, this technology needs to use a lot of equipment, and it pays special attention to fast, accurate and application. The working platform of food detection technology is microbial detection instrument, which has the characteristics of miniaturization, function specialization, integration, low energy and imaging. Food microbiological detection technology can be integrated with the achievements of biotechnology. Nucleic acid probe technology and PCR technology are widely used in microbiological inspection. Even though food 
microbiological detection technology has certain application in food safety detection, many technologies have not been used in food safety detection. For example, biochip technology and biosensor have not been well used. Therefore, if the future of food safety testing to use microbial detection technology, then there is still a process of exploration, to continue to develop and improve. For example, the problems of contamination and false positive can be solved by hybridization of nucleic acid probe and PCR. In the future, online nondestructive food microbiological testing technology should also be paid attention to, and then replace those destructive microbial detection methods, and non-destructive, in-situ detection of microbial detection technology will develop better and better. With the rapid development of science and technology, more and more microbial detection technologies will be applied to food detection and play an increasingly important role in the field of food safety.

\section{CONCLUSIONS}

Nowadays, with more and more food safety incidents, food microbiological detection is very important. And food microbiological detection is a key step in safety testing, therefore, the rapid detection technology of microorganisms is in urgent need of everyone's support. In our daily detection process, researchers can choose the most suitable method according to the detection environment and objectives, and can also combine several detection methods, so as to improve the detection efficiency, better serve the regulatory authorities, so as to ensure the food safety purchased by the public. to make a long story short, The important content of food safety detection includes the rapid and accurate detection of bacteria in food. The emergence of microbial detection technology has completely broken the limitations of traditional detection methods, and its advantages of high sensitivity, low detection cost, short detection time and simple operation, its effective use in food safety detection can not only meet the needs of rapid detection To a certain extent, it can also accurately get the test results, which has great application value.

\section{References}

1. Cao Keren, sun Rongrong, and Lin Xiaohui. "Research progress on rapid detection technology of food microorganisms." Chinese and foreign food industry: Xia ,2015, pp. 52 - 71. DOI: https://doi.org/10.1007/BFb0025774

2. Li Ying, "research progress on rapid detection technology of food pathogenic microorganisms", biochemistry,2017, pp. 337 - 351. DOI: https://doi.org/10.1007/3-540-11494-7_22

3. Wei Qingling. "Research on the content and technology of food microbiological test." clinical medical research and practice, 2016.

4. Chen Xuexia. "Research on the content and detection technology of food microbiological inspection." food safety guide,2017, pp. $220-270$. DOI: https://doi.org/10.1007/978-3-540-72522-0_6

5. Wang Dali. "Analysis of food microbiological inspection content and detection technology", China health nutrition,2015, pp. 53 - 113. DOI: https://doi.org/10.1007/978-3-642-21455-4_3

6. Xiang Wenjin, Xu Yuancong, and $\mathrm{Xu}$ Wentao. "Research progress on rapid detection of microorganisms in aquatic products and aquatic products." Chinese fisheries quality and standards,2016 pp. 214 - 229. DOI: https://doi.org/10.1007/978-3-540-30494-4_16

7. Jie Li, Chen Ling, and Wang Xiaopeng. "Exploration of food microbiological detection technology and quality control." food safety guide,2017, pp. 154 169. DOI: https://doi.org/10.1007/10722167_15

8. Li Xin. "Microbial detection technology and its application in food safety." family health,2020, pp. 51 - 63. DOI: https://doi.org/10.1145/800057.808665

9. Luo Yongliang. "Application and effect of different microbiological testing techniques in food safety." China health nutrition,2019, pp. 123 - 144. DOI: https://doi.org/10.1007/978-3-642-82453-1_5

10. Turhong hasmu, and Li Yong. "Application of the latest microbial technology in food safety detection." food safety guide 25(10) (2019) 40 - 51. DOI: https://doi.org/10.1109/2.161279 